\title{
Central motor conduction studies in hereditary spastic paraplegia
}

\author{
W Schady, J P R Dick, A Sheard, S Crampton
}

\begin{abstract}
Central motor conduction (CMC) studies were carried out in 25 patients with hereditary spastic paraplegia (HSP). Responses evoked in the lower limbs by transcranial magnetic stimulation of the motor cortex were bilaterally absent in $33 \%$ of the patients and, when recordable, were delayed in $75 \%$ of cases. Responses in the upper limbs were mostly normal except for those from the five members of one family, which were considerably delayed. There was no correlation between CMC parameters and age, duration of disease or upper limb hyperreflexia. CMC time to the tibialis anterior correlated with disability in patients with juvenile-onset HSP. It is concluded that CMC studies are not useful in detecting subclinical lesions in hereditary spastic paraplegia but may be of value in identifying subgroups of the disease.
\end{abstract}

Hereditary spastic paraplegia (HSP) was first described by Strümpell. ${ }^{1}$ It is usually inherited as an autosomal dominant trait, although in some kinships transmission may be autosomal recessive. ${ }^{23}$ In most families the clinical picture is remarkably homogeneous, consisting of gradually progressive lower limb spasticity which is disproportionate to the degree of weakness. Upper limb tendon reflexes are sometimes exaggerated. Sensation is usually normal on bedside examination but subclinical impairment of cutaneous sensitivity is common. ${ }^{4}$ There are two types of HSP as defined by the age of onset. In type 1 HSP symptoms develop before the age of 35 , while in type 2 the onset of symptoms is delayed until after this age in most family members. ${ }^{3}$ In the authors' series 20 years was found to be a better cut-off point between the two types of the disease. ${ }^{4}$ There are also complex forms of HSP, where lower limb spasticity is associated with extrapyramidal features, optic atrophy, cerebellar ataxia or a sensory neuropathy.

Investigation of patients with HSP is usually unrewarding and is carried out mainly to exclude other causes of cord disease such as multiple sclerosis or cervical spondylotic myelopathy. Visual evoked responses and the CSF are normal, although somatosensory evoked potentials may be small or delayed in a proportion of patients. ${ }^{47}$ Little information is available on conduction in central motor path- ways in HSP. This can be assessed by transcranial electrical $^{8}$ or magnetic ${ }^{9}$ brain stimulation, though the latter is preferred because it is painless. We have studied central motor conduction in HSP using magnetic brain stimulation to ascertain whether there are distinctive abnormalities which might help to differentiate this disorder from other common cord syndromes. ${ }^{10-15}$

\section{Materials and methods PATIENTS}

Twenty five patients from 17 kinships with hereditary spastic paraplegia were studied. The diagnosis was made on the basis of a welldocumented family history, characteristic clinical findings and gradually progressive evolution. No sporadic cases were accepted for study. Inheritance was autosomal dominant in 15 families. In two families siblings were affected but there was no history of either parent having had the disease, indicating either autosomal recessive inheritance or variable clinical expression in a dominantly inherited disorder. The age of onset was uniformly under 20 years in 11 patients from seven families (type 1). In nine other families it was uniformly greater than 20 (type 2), while in the three members of one family the age of onset was 15, 16 and 25 years. For the purpose of comparisons between groups the latter intermediate family was considered to be of type 1 .

Fifteen patients were male and 10 were female. Their ages ranged from 16 to 73 years (mean 45 years). Though the clinical picture was dominated by a spastic paraparesis, all patients were still mobile. Brisk upper limb reflexes were found in 13. Six patients had evidence of sensory impairment on standard bedside examination and most of the others had abnormal results on quantitative sensory testing. This has been described elsewhere. ${ }^{4}$ No patient had amyotrophy, deafness, intellectual impairment, skin changes, retinal pigmentation, cerebellar ataxia or clinical evidence of extrapyramidal disease. Five members of one family had reduced or absent sensory action potentials in upper and lower limbs. The remainder of the patients had normal nerve conduction studies. The severity of the motor deficit was assessed by the Functional Disability Index, ${ }^{16}$ where $1=$ minimal impairment, $2=$ mild weakness, 3 = moderate weakness, $4=$ moderate impairment of gait, $5=$ marked impairment, 6 = stick needed, $7=$ walking frame needed, 
Figure 1 Relationship between disability grade (see text) and duration of disease (in years) for patients with juvenileonset HSP (type 1, filled circles), adult-onset $H S P$ (type 2, open circles) and the intermediate form (triangles). The slope of the regression line is steeper for type 2 $(r=0.73, p<0.05)$ than for type 1 and intermediate $(r=0.58$, $p<0.05$ ).

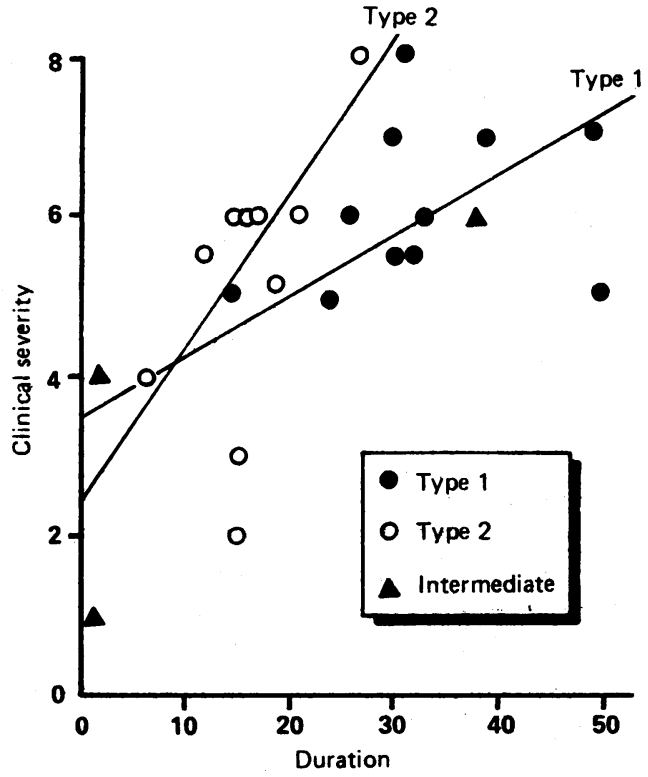

$8=$ crutches needed. Higher grades did not apply. The duration of symptoms varied from 1 to 50 years. The degree of disability correlated with the duration of disease both for type 1 and type 2 cases, though in the latter (adult-onset) group the disease progressed more rapidly (fig 1 ).

CONTROLS

Normative data were obtained from 30 healthy controls of both sexes aged 21 to 69 years (mean 44 years). They were medical or paramedical personnel without any history of nervous system disease.

\section{CENTRAL MOTOR CONDUCTION STUDIES}

The motor cortex was activated with a magnetic stimulator (Magstim, UK) consisting of a capacitor which is rapidly discharged through a circular copper coil $9 \mathrm{~cm}$ in mean diameter. This was placed on the scalp with its centre at the vertex. When recording from the lower limbs better responses were sometimes obtained if the stimulating coil was positioned a little to one side of the midline. During cortical stimulation subjects were instructed to maintain a slight contraction (10-15\% of maximum) of the muscle from which responses were being recorded. None of the patients was too weak to contract the relevant muscles. The stimulus intensity was gradually increased to $80-100 \%$ of the maximum output of the device (2 Tesla). By inverting the coil it was possible to activate preferentially the motor cortex of one or other hemisphere.

Surface recordings were obtained with silver/silver chloride cup electrodes and a Cadwell 7400 electrodiagnostic system. At least five responses were obtained from the abductor digiti minimi (ADM), abductor pollicis brevis (APB) and tibialis anterior (TA) muscles on both sides. The following measurements were made: latency to the onset of the earliest compound muscle action potential, amplitude of the largest negative peak and mean duration of the CMAP. Responses were not averaged.
Recordings were always made from right and left limbs.

Electrical stimulation of the ulnar and median nerves at the wrist and of the common peroneal nerve at the knee were carried out using standard techniques. The recording points were the same as for magnetic stimulation of the cortex. The latency and amplitude of a supramaximal $M$ response and the shortest latency of at least $10 \mathrm{~F}$ waves were measured. The central motor conduction (CMC) time was obtained by subtracting $(F+M-1) / 2$ from the latency of the response evoked by magnetic stimulation of the cortex. The amplitude of cortical responses was expressed as a percentage of the maximal $M$ wave resulting from distal nerve stimulation. This adjustment was necessary because, although mean (SD) upper limb $M$ wave amplitudes were similar in patients and in controls, those from the tibialis anterior were smaller in patients $[2.9(1.1) \mathrm{mV}]$ than in the control population $[4 \cdot 1(2 \cdot 1) \mathrm{mV}]$.

\section{ANALYSIS OF DATA}

CMC times in the control population were normally distributed. Amplitude ratios in controls were log transformed to obtain a normal distribution. Parametric statistics were used throughout, except for comparisons involving disability grades, when Spearman's correlation coefficient was used.

\section{Results}

CONTROL SUBJECTS

Compound muscle action potentials in response to magnetic stimulation of the motor cortex could be readily obtained from ADM and APB on both sides. Responses from the TA were also recordable bilaterally in all but five subjects, in whom they were clearly recognisable on only one side. This represents a failure rate in the TA of $9 \%$. The amplitude of cortically evoked responses ranged from 1.8 to $10.3 \mathrm{mV}$ in APB, from 1.2 to $10.0 \mathrm{mV}$ in $\mathrm{ADM}$ and from 0 to $5 \cdot 1 \mathrm{mV}$ in the TA. The minimum normal value, mean (2.5 SD), of cortical to distally evoked CMAP amplitude ratios was $16 \%$ for APB and $10 \%$ for ADM. Responses to TA were only considered abnormal by amplitude criteria when bilaterally absent. There was no correlation of response amplitude with height, age or gender.

There was far less variability in latency than in amplitude of cortically evoked responses. Height, arm length and gender had no significant effect on CMC time. The right and left sides were not significantly different for response amplitude or latency. The maximum right-left difference in CMC time was $2.6 \mathrm{~ms}$ for APB, $1.9 \mathrm{~ms}$ for ADM and $2.8 \mathrm{~ms}$ for TA. There was a correlation with age for CMC time to APB $(r=0.46, p<0.001)$ and TA ( $r=0.64, p<0.001$ ) but not to ADM $(r=0.24, \mathrm{NS})$. Corresponding linear regression equations were $\mathrm{y}=4.8+0.04 \mathrm{X}$ (APB), $y=8.3+0.09 X$ (TA) and $y=5.3+$ 0.015X (ADM). Patients' CMC times were considered abnormal if they fell outside the $95 \%$ confidence limits of the regression line. 
Table Range of central motor conduction times in healthy subjects and in patients with HSP (in ms). Values for members of family 4 were very prolonged and are given separately.

\begin{tabular}{llcr}
\hline & & \multicolumn{3}{c}{ HSP } \\
& Control & (except family 4) & Family 4 \\
\hline APB & $4 \cdot 4-8 \cdot 9$ & $4 \cdot 4-9 \cdot 1$ & $11 \cdot 9-18 \cdot 3$ \\
ADM & $4 \cdot 3-8 \cdot 4$ & $4 \cdot 2-10 \cdot 6$ & $7 \cdot 6-16.9$ \\
TA & $9 \cdot 0-16 \cdot 2$ & $10 \cdot 7-36 \cdot 1$ & $30 \cdot 1-39 \cdot 3$ \\
\hline APB $=$ & abdur
\end{tabular}

APB = abductor pollicis brevis. $\mathbf{A D M}=$ abductor digiti minimi. $\mathrm{TA}=$ tibialis anterior.

\section{PATIENTS}

Peripheral motor conduction times, measured by using the formula $(F+M-1) / 2$, were not significantly different in patients and control subjects, whether recording from APB, ADM or TA.

CMAPs in response to cortical stimulation could be obtained from the hand muscles in all patients, though in two cases they were readily reproducible on one side only. In three other patients responses from one hand were reduced in amplitude. With regard to the lower limbs, cortically evoked responses from the TA were bilaterally absent in eight patients. Most of the remainder were at the lower end of the normal spectrum (mean $25 \%$ against $35 \%$ for healthy controls).

Central motor conduction times to APB, $A D M$ and TA were considerably abnormal in the five members of one family, whose values amounted to about twice those of other patients (table). The amplitudes of their corticallyevoked responses, however, were no different from those of the rest of the patients (fig 2). These findings prompted a review of the clinical features of members of family 4 , since they suggested that the underlying pathological process was not the same. The history and physical findings were comparable to those of other patients with hereditary spastic paraplegia. They had a slowly progressive syndrome of lower limb spasticity beginning in the first or second decade, with moderate leg weakness and hyperreflexia of upper and lower extremities. Disease duration was 30-39 years. The only abnormality on investigation was reduction or absence of peripheral sensory action potentials.

Apart from the five members of family 4, six

Figure 2 Representative examples of cortically evoked motor responses from the tibialis anterior in a normal subject, a patient with $H S P$ and a member of family 4. Two responses are superimposed.

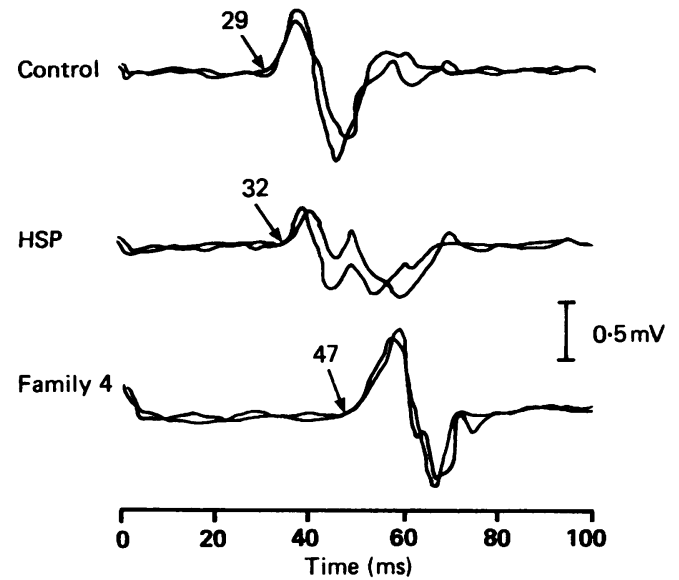

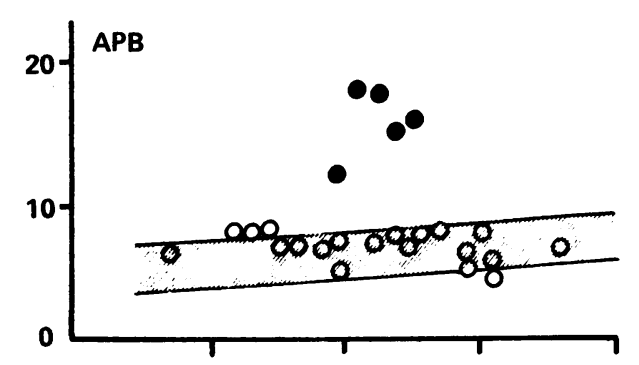
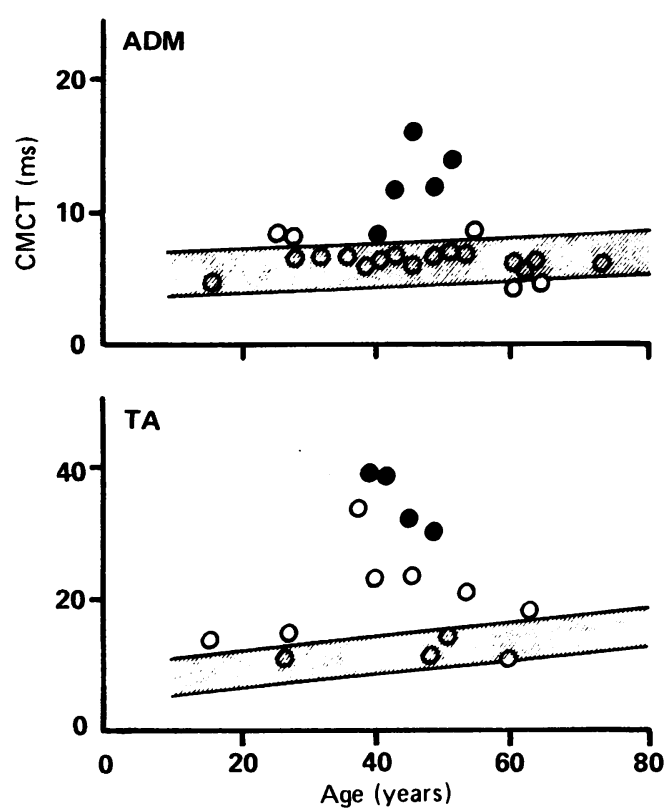

Figure 3 Central motor conduction time (CMCT) in patients with hereditary spastic paraplegia against age. The upper panel shows results obtained from abductor pollicis brevis $(A P B)$, the middle panel from abductor digiti minimi (ADM), and the lower panel from tibialis anterior (TA). The shaded area corresponds to the normal range. Members of family 4 are represented by filled circles, other patients by open circles. Only the shortest latency from either side is given.

patients had marginally prolonged CMC times to the hand muscles, in two cases to both APB and $\mathrm{ADM}$. Abnormalities were more evident in the lower limbs (fig 3). Twelve of the patients who had recordable responses over the TA showed a delay of central conduction at this site, sometimes to a marked degree. Only four patients $(17 \%)$ showed normal corticallyevoked responses from the TA in terms of both latency and amplitude. The duration of the responses was not a useful parameter. The mean (SD) duration of potentials recorded from the TA was $25 \cdot 8(8 \cdot 8) \mathrm{ms}$ in HSP patients against $24 \cdot 1(4 \cdot 2) \mathrm{ms}$ in controls.

Cortical motor response variables were compared with certain clinical features. CMC time and response amplitudes were no different in type 1 HSP patients, whose symptoms developed before the age of 20 , from those in type 2 patients, who presented later. Equally, there was no correlation between cortical response parameters and the patient's age or disease duration. CMC time to the TA correlated weakly with disability in patients with type $1 \mathrm{HSP}(r=0.64, p<0.05)$ but not in those with type 2 . Of the 13 patients with some abnormality of CMC to the hand muscles, eight had brisk upper limb reflexes. Five other patients with similarly brisk arm tendon 
reflexes had normal CMC to the upper extremities.

\section{Discussion}

Magnetic stimulation of the cortex is a useful technique for assessment of the integrity of motor pathways. ${ }^{9}$ It has displaced electrical cortical stimulation, which is often painful, and indirect techniques for measuring central motor conduction which rely on long latency reflex responses. ${ }^{17}$ The main disadvantages of transcranial cortical stimulation are variability in the response amplitude and lack of specificity of the recorded abnormalities, which may be similar in such disparate disorders as multiple sclerosis, motor neuron disease and cervical spondylotic myelopathy. ${ }^{11131518}$

To obtain the central motor conduction (CMC) time, the peripheral component has to be subtracted from the latency of the cortical response. We chose to do this by recording peripherally evoked $M$ and $F$ waves and employing the formula $(F+M-1) / 2 .{ }^{151920}$ The alternative, namely recording responses from electrical or magnetic stimulation over the vertebral column, has the drawback of incorporating a short length of the ventral roots in the CMC measurements. ${ }^{1921}$ The $\mathrm{F}$ wave method should provide an adequate measure of peripheral motor conduction time as long as the fastest $F$ and $M$ responses are mediated by the same fibres. In normal subjects there is general agreement that this is the case. ${ }^{223}$ Since peripheral motor conduction times in our patients were no different from those of controls, it can be assumed that the normal relationship between $F$ and $M$ latencies holds in HSP.

As with previous authors, we have expressed the amplitudes of cortical responses as a percentage of the maximal CMAP evoked by peripheral nerve stimulation, a particularly important precaution in studies of heredodegenerative disorders, where there may be an associated neuropathy. Some loss of motor axons in the lower limb nerves of our patients is suggested by the smaller size of compound muscle action potentials from the tibialis anterior than in age-matched controls. This would have resulted in a spurious reduction of cortically evoked responses if absolute values had been employed.

Hereditary spastic paraplegia (HSP) is an ideal disorder to study by transcranial motor stimulation. Other investigations are usually negative and in the early stages it is often misdiagnosed as multiple sclerosis. Two reports refer to central motor conduction in HSP. Thompson et $a l^{24}$ described two cases whose responses, obtained by electrical stimulation of the cortex, were normal in amplitude but asymmetrically delayed. Claus et $a l^{25}$ reported abnormalities in CMC time to the upper extremities in two out of 10 patients. CMC times to the lower limbs were prolonged in all four patients in whom recordings from the tibialis anterior were obtained.

Our results in a larger group of patients clearly indicate that the lower limbs are more likely to yield abnormal results in HSP. Cen- trally evoked CMAPs in the tibialis anterior were bilaterally absent in a third of patients and were delayed in three quarters of the patients who had recordable responses. By amplitude or latency criteria $83 \%$ of lower limb responses were abnormal. In most patients the potentials recorded from the upper limbs were normal or only marginally deranged. This underlines the clinical and histological evidence that the bulk of the pathology in HSP is in the lower spinal cord. In Strümpell's cases the lesions were virtually confined to the lateral pyramidal tracts, increasing from cervical to lumbar region. ${ }^{26}$ Schwarz and $\mathrm{Liu}^{27}$ reported that demyelination and loss of axons were symmetrical and most extensive in the thoracic cord. Behan and Maia ${ }^{28}$ also found that degeneration of the corticospinal tracts became progressively more severe at lower levels.

Central motor conduction results did not discriminate between patients with normal and exaggerated upper limb tendon reflexes. While almost two thirds of the patients with abnormal CMC times to the hand muscles had brisk upper limb reflexes, arm hyperreflexia was not a good predictor of deranged CMC. At first glance, this suggests that the technique lacks sensitivity, at least in the context of HSP. In multiple sclerosis, on the other hand, subclinical lesions in the cervical cord may be uncovered by CMC studies. ${ }^{11}$ The incidence of upper limb CMC abnormalities is also higher in tropical spastic paraparesis than in HSP. ${ }^{29}$ With regard to other inherited neurodegenerative diseases, CMC to hand muscles is abnormal in the great majority of patients with Friedreich's ataxia and early onset cerebellar ataxia. $^{14}$ It is not clear why the technique should be more sensitive to upper limb motor dysfunction in these disorders than in HSP. Differences in the distribution of the spinal cord lesions may be responsible. Alternatively, the time course of the disease may have a bearing on CMC values. HSP is a very slowly progressive disorder by comparison with most cases of multiple sclerosis. It is possible that this allows sprouting of pyramidal tract neurons which is sufficient to normalise the CMC parameters even in the presence of upper limb hyperreflexia.

One family stands out as being different from the rest. All members of kinship 4 had marked prolongation of CMC time to upper and lower limbs, averaging twice that seen in the other patients. This indicates slowing of central conduction compatible with demyelination, whereas the results in other patients (at least those in the upper extremities) could be explained on the basis of selective loss of axons in the corticospinal tract, with resulting increase in the time needed for spinal motor neurons to reach the firing threshold. Family 4 is further unusual as all tested siblings had reduced or absent peripheral sensory action potentials. The history and examination findings, however, were indistinguishable from those of other patients with HSP and were quite different from those of cases of so-called hereditary sensory neuropathy with spastic paraplegia. ${ }^{30}$ 
We found no correlations between CMC parameters and age of onset or disease duration. While it might be argued that the very increased CMC times observed in members of family 4 related to the considerable length of their disease (30-39 years), there were two other patients with even greater disease duration whose upper limb responses were normal in latency. As expected, disability increased with age, progression being more rapid in patients with adult-onset HSP (type 2). Surprisingly, in this subgroup there was no correlation between degree of disability and CMC time, though such a correlation did exist for type 1 patients.

In conclusion, $\mathrm{CMC}$ studies have not proved to be of value in detecting subclinical lesions in most patients with HSP. Therefore it is unlikely that the technique will be useful in the early detection of affected family members. From the results of our study, the most valuable contribution of transcranial motor stimulation in HSP is to help identify subgroups of patients whose results differ from the norm. It is possible that the underlying biochemical defect will also be different and that HSP will prove to be genetically heterogeneous.

We thank Drs CE Clarke, RG Lascelles, MA Sambrook and DI Shepherd for referring patients for study.

1 Strümpell A. Beiträge zur Patholgie des Rückenmarks. Arch Psychiatr Nervenkr 1880;10:676-717.

2 Holmes GL, Schaywitz BA. Strümpell's pure familia spastic paraplegia: case study and review of the literature. J Neurol Neurosurg Psychiatry 1977;40:1003-8.

3 Harding AE. Hereditary "pure" spastic paraplegia: a clinical and genetic study of 22 families. $J$ Neurol Neurosurg Psychiatry 1981;44:871-83.

4 Schady W, Sheard A. A quantitative study of sensory function in hereditary spastic paraplegia. Brain 1990;113:709-20.

5 Pedersen L, Trojaborg W. Visual, auditory and somatosensory pathway involvement in hereditary cerebellar ataxia Friedreich's ataxia and familial spastic paraplegia. Electroenceph Clin Neurophysiol 1981;52:283-97

6 Thomas PK, Jefferys GJR, Smith IS, Loulakakis D. Spinal somatosensory evoked potentials in hereditary spastic paraplegia. J Neurol Neurosurg Psychiatry 1981;44:243-6.

7 Dimitrijevic MR, Lenman JAR, Prevec T, Wheatly K. A study of posterior column function in familial spastic paraplegia. J Neurol Neurosurg Psychiatry 1982;45:46-9.

8 Merton PA, Morton HB. Stimulation of the cerebral cortex in the intact human subject. Nature 1980;285:227.
9 Barker AT, Jalinous R, Freeston IL. Non-invasive magnetic stimulation of human motor cortex. Lancet 1985;ii: 1106-7.

10 Hess CW, Mills KR, Murray NMF. Measurement of central motor conduction in multiple sclerosis by magnetic brain stimulation. Lancet 1986;ii:355-8.

11 Hess CW, Mills KR, Murray NMF, Schreider TN. Magnetic brain stimulation: central motor conduction studies in multiple sclerosis. Ann Neurol 1987;22:744-52.

12 Ingram DA, Thompson AJ, Swash M. Central motor conduction in multiple sclerosis: evaluation of abnormalities revealed by transcutaneous magnetic stimulation malities revealed by transcutaneous magnetic stimulation of the

13 Thompsen PD, Dick JPR, Asselman P, et al. Examination of motor function in lesions of the spinal cord by stimulation of the motor cortex. Ann Neurol 1987;21:389-96.

14 Claus D, Harding AE, Hess CW, Mills KR, Murray NMF, Thomas PK. Central motor conduction in degenerative ataxic disorders: a magnetic stimulation study. $J$ Neurol Neurosurg Psychiatry 1988;51:790-5.

15 Eisen A, Shytbel W, Murphy K, Hoirch M. Cortical magnetic stimulation in amyotrophic lateral sclerosis. Muscle Nerve 1990;13:146-51.

16 Donofrio PD, Tandan R, Albers JW. Plasma exchange in chronic inflammatory demyelinating polyradiculochronic inflammatory demyelinating

17 Eisen A, Hoirch M, Fink M, Goya T, Calne D. Noninvasive measurement of central sensory and motor conduction. measurement of central sens

18 Ingram DA, Swash M. Central motor conduction is abnormal in motor neurone disease. J Neurol Neurosurg Psychiatry 1987;50:159-66

19 Ugawa Y, Rothwell JC, Day BL, Thompson PD, Marsden CD. Magnetic stimulation over the spinal enlargements $J$ Neurol Neurosurg Psychiatry 1989;52:1025-32.

20 Berger AR, Shahani BT. Electrophysiologic evaluation of spinal cord motor conduction. Muscle Nerve 1989;12: 976-80.

21 Mills KR, Murray NMF. Electrical stimulation over the human vertebral column: which neural elements are excited? Electroenceph Clin Neurophysiol 1986;63:582-9.

22 Kimura J. F-wave velocity in the central segment of the median and ulnar nerves. A study in normal subjects and in patients with Charcot-Marie-Tooth disease. Neurology

23 Shahani BT, McLeod WN, Bertics GM. Minimal F response latencies as a measure of conduction in largestdiameter alpha-motor axons. Neurology /NY 1987;3:114

24 Thompson PD, Day BL, Rothwell JC, et al. The interpretation of electromyographic responses to electrical stimulation of the motor cortex in diseases of the upper motor neurone. J Neurol Sci 1987;80:91-110.

25 Claus D, Waddy HM, Harding AE, Murray NMF, Thoma PK. Hereditary motor and sensory neuropathies and Pereditary spastic paraplegia: a magnetic stimulation study. Ann Neurol 1990;28:43-9.

26 Strümpell A Die primäre Seitenstragsklerose (spastische Spinalparalyse). Dtsch Z Nervenheilkunde 1904;27: 291-339.

27 Schwarz GA, Liu CN. Hereditary (familial) spastic paraplegia. Further clinical and pathologic observations. Arch Neurol 1956;75:144-62.

28 Behan WMH, Maia M. Strümpell's familial spastic paraplegia: genetics and neuropathology. $J$ Neurol Neurosurg Psychiatry 1974;37:8-20.

29 Waddy H, Claus D, Murray NMF, Rudge P. Central moto conduction studies in tropical spastic paraparesis (TSP). Electroenceph Clin Neurophysiol 1990;75:S159-60.

30 Cavanagh NPC, Eames RA, Galvin RJ, Brett EM, Kelly RE. Hereditary sensory neuropathy with spastic paraplegia. Brain 1979;102:79-94. 